\begin{tabular}{ll|l|l|l|l|}
\hline Crystal Res. Technol. & $\mathbf{1 9}$ & 1984 & 7 & $\mathrm{~K} 6 \mathrm{l}-\overline{\mathrm{K} 63}$
\end{tabular}

\author{
Short Notes \\ P. Gille, P. Rudolph \\ Humboldt-Universität zu Berlin, Sektion Physik, Bereich Kristallographie
}

\title{
On a Possibility of Avoiding Axial Segregation in Growing Pseudobinary Alloys from a Liquid Zone
}

There is considerable interest in macroscopically homogeneous crystals in a great variety of pseudobinary systems $\mathrm{A}_{1-x} \mathrm{~B}_{x} \mathrm{C}$ (e.g. $\mathrm{Hg}_{1-x} \mathrm{Cd}_{x} \mathrm{Te} ; \mathrm{Pb}_{1-x} \mathrm{Sn}_{x} \mathrm{Te}$ ). Crystal growth methods from a liquid zone, i.e., zone melting and similar techniques (e.g. travelling heater method - THM), are known to provide with crystalline ingots being fairly uniform in composition, except for the initial part of the crystal. For a binary system the solute distribution is described by the well-known zone melting function (Pfann): $:^{1}$ )

$$
x_{\mathrm{S}}(z)=x_{0}\left[1-\left(1-k_{1}\right) \exp \left(-\frac{z}{l} k_{1}\right)\right] .
$$

Besides a complete mixing of the melt and other prerequisites a homogeneous composition $x_{0}$ of the source material is demanded which is identical with the zone composition at the very beginning $x_{\mathrm{L}}(0)=x_{0}$. Just this original concentration is the cause of the initial part of the distribution function providing an inhomogeneous first-togrow region until reaching steady-state conditions $\left(x_{\mathrm{L}}=x_{0} / k_{1}\right)$ within the liquid zone.

DibenedetTo, Cronan mentioned the possibility of avoiding the initial course in a zone crystallization distribution by selecting the suitable zone composition by means of trial and error. Nevertheless, most workers dealing with problems of THM crystal growth of solid alloys use a pure solvent zone at the very beginning of the growth run to dissolve solid material at the interfaces. (e.g. WolfF et al.; Triboulet, Didier; Kobayashi, Takagi).

It is the intention of this note to give the expression for an appropriate zone composition that avoids the initial course of the distribution function, which usually takes a considerable portion of the grown crystal.

In principle, it is possible to start with a separately prepared zone material of just the steady-state composition $x_{\mathrm{L}}(0)=x_{0} / k_{1}$. This idea seems to become even more important if a method is used, which (i) necessitates in any case a separately prepared zone material, and (ii) provides crystals being not much longer than some few zone lengths $(L / l \lesssim 10)$, i.e., which not much exceed the initial part of PFanN's zone melting distribution. The travelling heater method must be considered such a technique.

Besides the mentioned distribution coefficient $k_{1}=x_{\mathrm{S}} / x_{\mathrm{L}}$ in the pseudobinary system describing the $\mathrm{A} / \mathrm{B}$ ratio of the alloy, another coefficient $k_{2}=y_{\mathrm{s}} / y_{\mathrm{L}}=1 / 2 y_{\mathrm{L}}$ at the section $x=$ constant within the ternary system becomes interesting. It is responsible for the THM principle (as well as for solution growth, in general) and describes the solubility with respect to an either $(A, B)$ or $C$ excess in the liquid zone

$\left.{ }^{1}\right)$ For the purpose of this study compositions are deseribed in terms of mole fractions. 


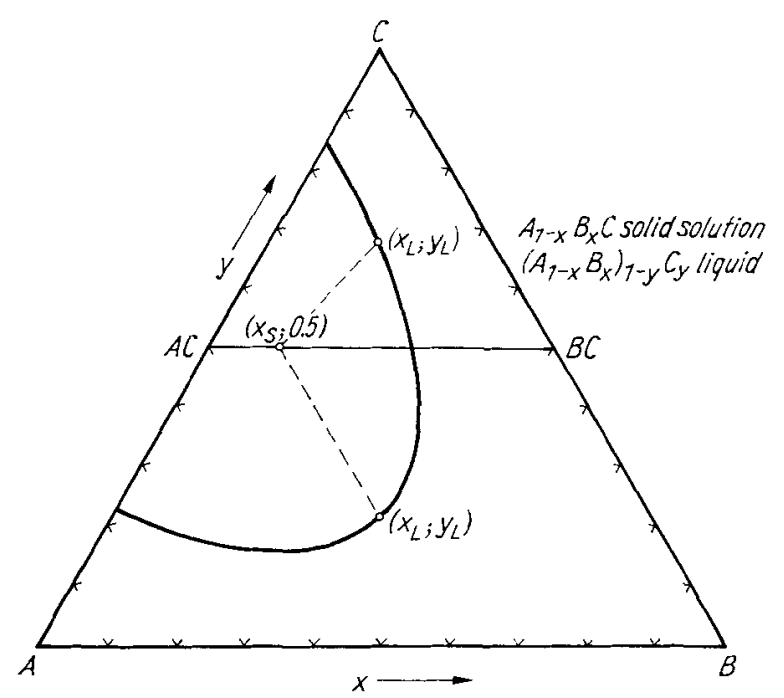

Fig. 1. Isothermal section through a ternary phase diagram involving the pseudobinary $\mathrm{A1}-x \mathrm{~B} x \mathrm{C}$ solid solution. Dashed lines mark the equilibria between a solid alloy with an (A, $\mathrm{B}$ ). rich or C-rich liquid phase

(see Fig. 1). Since $y_{\mathrm{S}}=0.5$ is fixed in the pseudobinary alloy $\mathrm{A}_{1-x} \mathrm{~B}_{x} \mathrm{C}, k_{2}$ is not regarded for the aim of this discussion. Deviations from the stoichiometry may be neglected. Nevertheless, the solving process, i.e., the equilibrium temperature will usually change the value of $k_{1}$ with respect to that in the pseudobinary system. Generally, the additional degree of freedom in solution growth allows the independent choice of $x_{\mathrm{L}}(0)$ without restricting the solving process.

Already PFANN described the method of doping an ingot during zone melting by only putting the dopant into the original zone, i.e., by allowing the initial zone composition $x_{L}(0)$ to differ from that of the source material. In such a way the distribution function (1) changes to:

$$
x_{\mathrm{S}}(z)=x_{0}-\left(x_{0}-x_{\mathrm{L}}(0) k_{1}\right) \exp \left(-\frac{z}{l} k_{1}\right) .
$$

Since THM in any case needs a separately prepared zone material it should be tried to reach such an $\mathrm{A} / \mathrm{B}$ ratio that after dissolving the feed and seed, respectively, an original liquid zone composition $x_{L}(0)=x_{0} / k_{1}$ will be obtained. It necessitates the exact knowledge of the distribution coefficient $k_{1}$ and the solubility $y_{L}$ at the temperatures used. Starting with the steady-state composition equation (2) runs to:

$$
x_{\mathrm{S}}(z)=x_{0}=\text { constant },
$$

thus providing a homogeneously axial distribution of the growing crystal from the very beginning, except for the last freezed zone length like in each zone crystallization. The comparison between the usual zone melting distribution and that resulting from an appropriate choice of the original zone composition in THM is to be seen from Figure 2. The macroscopically homogeneous feed which is necessary for maintaining steady-state conditions is to be achieved by either quenching a liquid alloy of a corresponding composition or sintering powdered solid mixtures which depends on the system under consideration.

Since there is no restriction with respect to either (A, B)-rich or C-rich solutions the possibility to grow homogeneous solid alloys by the travelling heater method de- 
scribed in this note may be used in all THM experiments from a native solvent known in the literature of a great variety of systems.

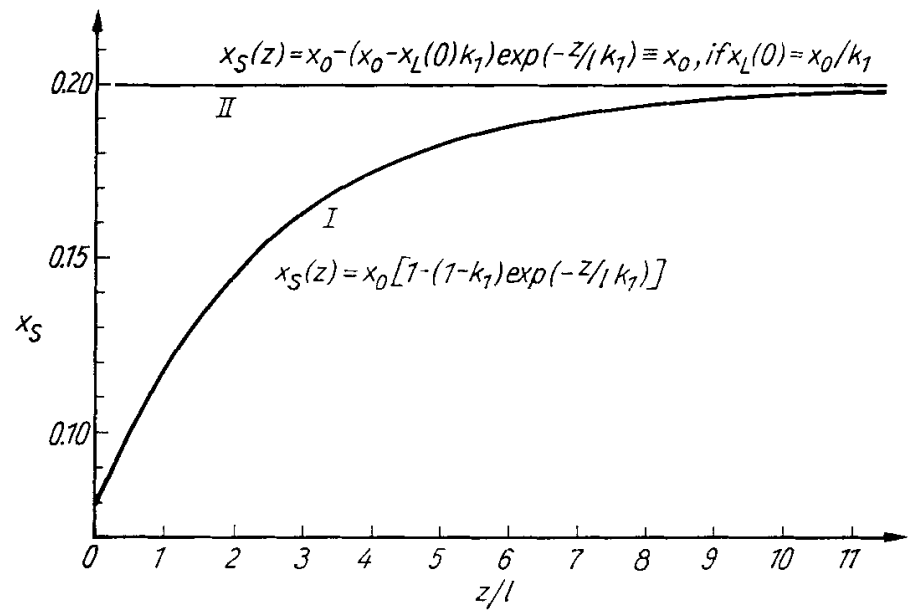

Fig. 2. Segregation in the psendobinary solid solution $\Lambda_{1}-x \mathrm{~B}_{x} \mathrm{C}$ grown from a liquid zone with $x_{0}(\mathrm{I})$ and from a liquid zone with an original composition $x_{0} / k_{1}$ (II). $x_{0}=0,2, k_{1}=0.4$

\section{Symbols}

$x_{\mathrm{L}}$ and $x_{\mathrm{S}}$ mole fraction $\mathrm{BC}$ of the liquid and solid, respectively, in the pseudobinary $\mathrm{AC}-\mathrm{BC}$ system

$y_{\mathrm{L}} \quad$ mole fraction $\mathrm{C}$ in the ternary liquid solution

$z \quad$ freezed length of the growing ingot

l. length of the liquid zone, assumed to be constant

$L \quad$ total length of the ingot

$k_{1} \quad$ distribution coefficient $k_{1}=x_{\mathrm{S}} / x_{\mathrm{L}}$, describing the $\mathrm{A} / \mathrm{B}$ ratio

$k_{2} \quad$ distribution coefficient $k_{2}=1 / 2 y \mathrm{~L}$, describing the $(\mathrm{A}, \mathrm{B}) / \mathrm{C}$ ratio

$x_{0} \quad$ mole fraction of the original ingot (source)

$x_{L}(0)$ mole fraction with respect to the $A / B$ ratio of the liquid zone at the very beginning.

\section{References}

Dibenedetto, B., Cronan, C. J.: J. Amer. Ceram. Soc. 51, 364 (1968)

Kobayashi, T., Takagi, K.: J. Cryst. Growth 62, 189 (1983)

Pran , W. G.: Zone Melting, New York 1958

Trtboulet, R., Didier, G.: J. Cryst. Growth 52, 614 (1981)

Wolff, G. A., Labelle, H. E. Jr., Das, B. N.: Trans. Met. Soe. AIME 242, 436 (1968)

(Received November 29, 1983)

P. GILLE, Doz. Dr. se. P. RudOLPH

Humboldt-Universität zu Berlin, Sektion Physik, Bereich Kristallographie

DDR-1040 Berlin

Invalidenstr. 43 